\title{
Theory of Anodic Stripping Square Wave Voltammetry on Spherical Mercury Electrodes
}

\author{
Šebojka Komorsky-Lovrić and Milivoj Lovrić \\ Department of marine and environmental research, "Ruđer Bošković” Institute, 10000 Zagreb, Croatia
}

RECEIVED JUNE 6, 2014; REVISED OCTOBER 3, 2014; ACCEPTED NOVEMBER 18, 2014

\begin{abstract}
Relationships between dimensionless anodic stripping square-wave voltammetric net peak currents and the dimensionless inverse electrode radius are curves with two asymptotes that depend on the duration of accumulation. The theory applies to reversible reduction of amalgam forming ions on stationary macro and micro spherical and hemispherical mercury electrodes.
\end{abstract}

Keywords: square wave voltammetry, spherical electrodes, theory, microelectrode

\section{INTRODUCTION}

The contamination of aquatic ecosystem by metal ions can be monitored by networks of submersible probes. ${ }^{1-5}$ For trace metal analysis the technique that is very well suited is stripping voltammetry in combination with microelectrodes. ${ }^{6-10}$ These electrodes are characterized by high mass transport density, small double-layer capacitance, small ohmic drop and steady-state current response in unstirred solutions. ${ }^{11-15}$ Thus, in the preconcentration step of stripping technique no agitation of solution is necessary. $2,4,9,11,12$ The concentration of amalgam forming ions can be measured by anodic stripping voltammetry on mercury microelectrodes. ${ }^{16,17}$ The application of square-wave voltammetry in the stripping phase is particularly efficient. ${ }^{18}$ The theory of this technique is well developed for the stationary planar electrode covered by thin mercury film, ${ }^{19}$ but not for spherical electrodes because of the need to consider the diffusion within the mercury drop. ${ }^{20-22}$ This problem was investigated for the cathodic reaction, without the accumulation, in our previous paper. ${ }^{23}$ In this communication the dependence of anodic stripping square-wave voltammetric net peak current and peak potential on the electrode radius and frequency is investigated theoretically for the reversible reduction of amalgam forming ions.

\section{THE MODEL}

A reversible electrode reaction on mercury drop electrode is considered:

$$
\begin{aligned}
& \mathrm{Ox}^{n+}+n e^{-} \rightleftarrows \operatorname{Red}(\mathrm{Hg}) \\
& t=0 ; \quad r \geq r_{\mathrm{m}}: \quad c_{\mathrm{Ox}}=c_{\mathrm{Ox}}^{*}, \quad c_{\mathrm{Red}}=0 \\
& 0 \leq r<r_{\mathrm{m}}: \quad c_{\mathrm{Ox}}=0, \quad c_{\mathrm{Red}}=0 \\
& t>0 ; r \rightarrow \infty: \quad c_{\mathrm{Ox}} \rightarrow c_{\mathrm{Ox}}^{*} \\
& r=r_{\mathrm{m}}: \quad\left(c_{\mathrm{Ox}}\right)_{r=r_{\mathrm{m}}}=\left(c_{\mathrm{Red}}\right)_{r=r_{\mathrm{m}}} \exp (\varphi) \\
& \varphi=\frac{n F}{R T}\left(E-E^{\circ}\right) \\
& D\left(\frac{\partial c_{\mathrm{Ox}}}{\partial r}\right)_{r=r_{\mathrm{m}}}=-\frac{I}{n F S} \\
& D\left(\frac{\partial c_{\mathrm{Red}}}{\partial r}\right)_{r=r_{\mathrm{m}}}=-\frac{I}{n F S} \\
& r=0: \quad\left(\frac{\partial c_{\mathrm{Red}}}{\partial r}\right)_{r=0}=0
\end{aligned}
$$

The meanings of symbols are the following: $c_{\mathrm{Ox}}$ and $c_{\text {Red }}$ are concentrations of ions in the electrolyte and atoms in the mercury, respectively, $c_{\mathrm{Ox}}^{*}$ is the bulk concentration of ions, $E$ is electrode potential, $E^{\circ}$ is stand-

\footnotetext{
* Author to whom correspondence should be addressed. (E-mail: mlovric@irb.hr)
} 
ard potential of electrode reaction (1), $D$ is a common diffusion coefficient, $I$ is a current, $n$ is a number of electrons, $F$ is Faraday constant, $S$ is the electrode surface area and $r_{\mathrm{m}}$ is the radius of mercury electrode.

The mass transport is calculated by the Feldberg approximation. ${ }^{24}$ The simulation procedure is described in the previous paper. ${ }^{23}$ The results are reported as dimensionless current $\Phi=I\left(n F S c_{\mathrm{Ox}}^{*}\right)^{-1}(D f)^{-0.5}$. In square wave voltammetry the current is sampled at the end of each pulse and the difference between two subsequent samples is called the net response: $\Delta \Phi=\Phi_{\mathrm{f}}-\Phi_{\mathrm{b}}$. The forward, oxidative $\left(\Phi_{\mathrm{f}}\right)$ and the backward, reductive $\left(\Phi_{\mathrm{b}}\right)$ components of the net response are also reported as a function of the potential of staircase ramp. The responses depend on the pulse amplitude $E_{\mathrm{sw}}$, the potential step $d E$, the inverse value of dimensionless electrode radius $\rho=r_{\mathrm{m}}^{-1}(D / f)^{0.5}$ and the dimensionless parameter $k_{\text {acc }}$ that defines the relationship between the accumulation time and frequency: $k_{\mathrm{acc}}=50 t_{\mathrm{acc}} f$. In the simulation each pulse is divided into 25 time increments, so that $\Delta t=(50 f)^{-1}$ and $k_{\text {acc }}=t_{\text {acc }} / \Delta t$. The number of time increments determines the precision of simulation. ${ }^{25}$ The calculations were simplified by assuming that both ions and atoms have equal diffusion coefficients.

\section{RESULTS AND DISCUSSION}

In anodic stripping square wave voltammetry the starting potential is much lower than the standard potential, the scan direction is positive and the stripping scan is preceded by the accumulation period during which the electrode is charged to the starting potential. In our calculations this process was simulated by the diffusion controlled reduction at constant potential during several seconds. Two examples of stripping voltammograms are shown in Figure 1. If $\rho=0.03$ the net dimensionless peak current is 2.00 and peak potential is $-0.006 \mathrm{~V} v s$. $E^{\circ}$. The maximum and minimum of the oxidative and reductive components are 1.13 and -0.86 , respectively, and the potentials of these extremes are $-0.004 \mathrm{~V}$ and $-0.008 \mathrm{~V}$, respectively. For ten times higher value of $\rho$ the net peak current is 6.61 times bigger than the same current in Figure 1a. The maximum of oxidative component is enhanced 7.33 times and the minimum of reductive component is 5.77 times deeper comparing to the extremes of respective components in Figure 1a. The peak potential in Figure $1 \mathrm{~b}$ is $-0.074 \mathrm{~V}$ vs. $E^{\circ}$, which is 68 $\mathrm{mV}$ lower than in Figure 1a.

The influence of the parameter $k_{\text {acc }}$ on the concentration of amalgam at the end of accumulation period can be seen in Figure 2, for $r / r_{\mathrm{m}} \leq 1$. If the radius of electrode is $100 \mu \mathrm{m}$ and the diffusion coefficient is $9 \times 10^{-6} \mathrm{~cm}^{2} \mathrm{~s}^{-1}$, these profiles correspond to the real parameters $t_{\text {acc }} / \mathrm{s}=0.4$ (1), 2 (2) and 10 (3). The corresponding concentrations of reactant are shown for $r / r_{\mathrm{m}} \geq 1$ The gradients of the later curves at the electrode surface can be defined as functions of the bulk concentration of reactant and the distance $\delta$ that is called the diffusion layer thickness:

$$
\left(\frac{\partial c_{\mathrm{Ox}}}{\partial r}\right)_{r=r_{\mathrm{m}}}=\frac{c_{\mathrm{Ox}}^{*}}{\delta}
$$

This distance depends on the accumulation time and electrode radius. ${ }^{26}$ It is characterized by two limiting values: $\lim _{r_{\mathrm{m}} \rightarrow \infty} \delta=\sqrt{\pi D t_{\mathrm{acc}}}$ and $\lim _{t_{\mathrm{acc}} \rightarrow \infty} \delta=r_{\mathrm{m}}$. The second limit corresponds to the steady-state conditions. Figure 3 shows the dependence of dimensionless thickness of diffusion layer on the accumulation parameter $k_{\text {acc. }}$. The points were calculated by the simulation, using Eq. (10), and the line is theoretical
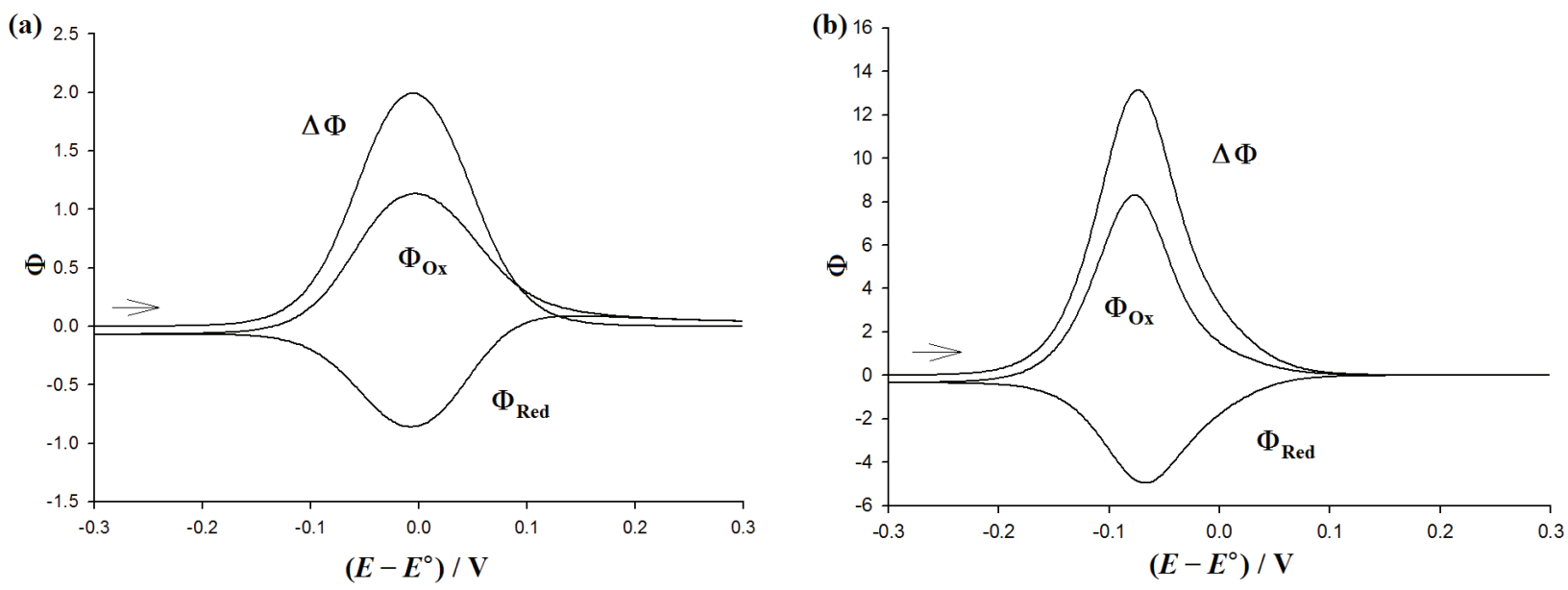

Figure 1. Anodic stripping square wave voltammograms of electrode reaction (1). $E_{\text {acc }}=-0.300 \mathrm{~V} v s . E^{\circ}, E_{\mathrm{st}}=E_{\text {acc }}$, $E_{\mathrm{sw}}=0.050 \mathrm{~V}, d E=0.002 \mathrm{~V}, k_{\mathrm{acc}}=1 \times 10^{4}$ and (a) $\rho=0.03$; (b) $\rho=0.30$. 


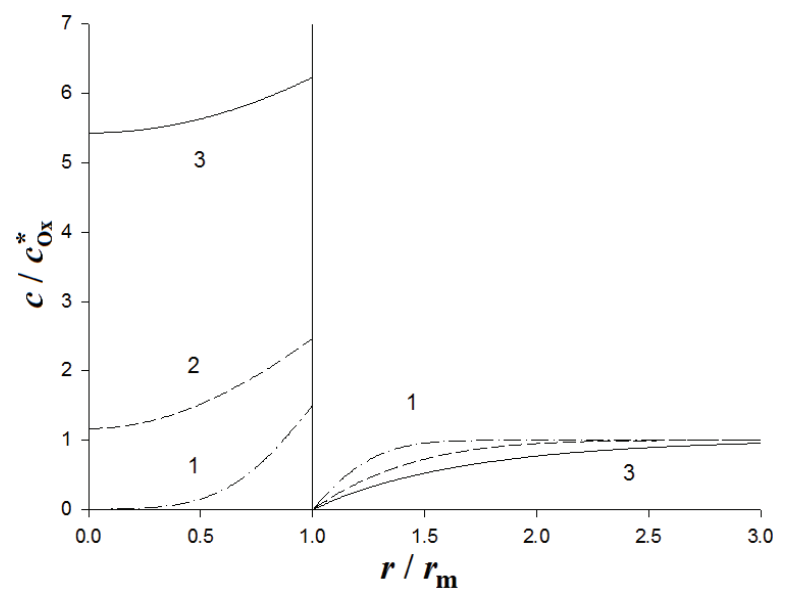

Figure 2. Relationship between relative concentrations of metal ions and amalgam at the end of accumulation period and the dimensionless distance from the center of spherical electrode. $E_{\text {acc }}=-0.300 \mathrm{~V}$ vs. $E^{\circ}, \rho=0.03$ and (1) $k_{\text {acc }}=2 \times 10^{3}$; (2) $k_{\text {acc }}=1 \times 10^{4}$; (3) $k_{\text {acc }}=5 \times 10^{4}$.

relationship:

$$
\frac{\delta}{\Delta r}=\frac{m \sqrt{\pi d k_{\mathrm{acc}}}}{m+\sqrt{\pi d k_{\mathrm{acc}}}}
$$

Here, $d=D \Delta t \Delta r^{-2}$ and $m$ is the number of space increments into which the electrode radius is divided $\left(r_{\mathrm{m}}=m \Delta r\right)$. The relationship (11) tends to the limit $m=$ 149 , which corresponds to $\rho=0.03$, but the tendency is rather slow for $k_{\mathrm{acc}}>10^{5}$ and the ratio $\delta / \Delta r$ becomes higher than $95 \%$ of this limit if $k_{\mathrm{acc}}>7.27 \times 10^{6}$. Because of relatively stable mass transport, the dependence of stripping peak current on the accumulation parameter can be approximated by the straight line $\Delta \Phi_{\mathrm{p}}=4.42 \times 10^{-5} k_{\mathrm{acc}}+2.37$. This is shown in Figure 4 .

Figure 5 shows the dependence of net peak current

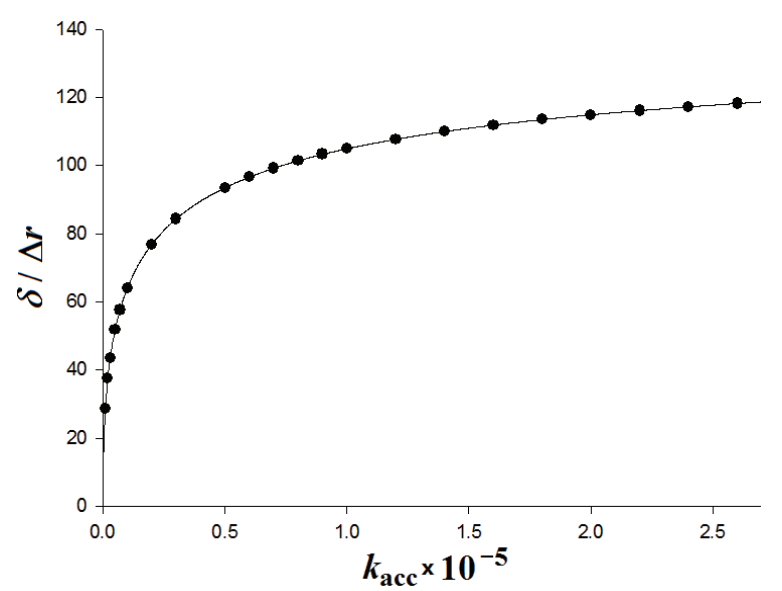

Figure 3. Relationship between dimensionless diffusion layer thickness and the accumulation parameter. All other data are as in Figure 2.

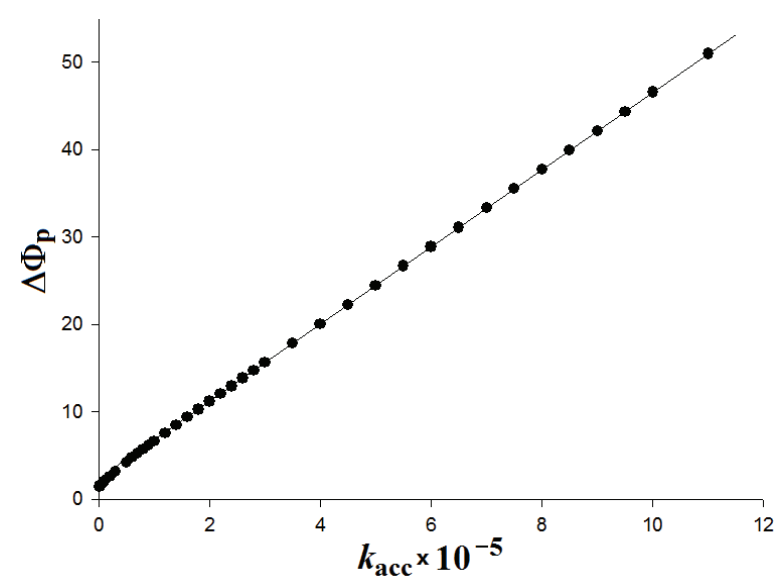

Figure 4. Relationship between dimensionless net peak current and the accumulation parameter; $\rho=0.03$ and all other data are as in Figure 1.

and peak potential on the parameter $\rho$. The relationships between $\Delta \Phi_{\mathrm{p}}$ and $\rho$ are curves that depend on the parameter $k_{\text {acc }}$. The straight lines in Figure $5 \mathrm{a}$ are asymptotes that are defined by the following equations: $\Delta \Phi_{\mathrm{p}}=35 \rho+0.5$, for $\rho<0.1$, and $\Delta \Phi_{\mathrm{p}}=10 \rho+4$, for $\rho>0.2$, both for $k_{\text {acc }}=2 \times 10^{3}, \Delta \Phi_{\mathrm{p}}=63.5 \rho+0.1$, for $\rho<0.1$, and $\Delta \Phi_{\mathrm{p}}=22.5 \rho+6.4$, for $\rho>0.2$, both for $k_{\text {acc }}=1 \times 10^{4}$, and $\Delta \Phi_{\mathrm{p}}=163.4 \rho$ for $k_{\text {acc }}=5 \times 10^{4}$. The conditions $\rho<0.1$ and $\rho>0.2$ mean that $r_{\mathrm{m}}>10 \sqrt{D / f}$ and $r_{\mathrm{m}}<5 \sqrt{D / f}$, respectively. These asymptotes show the relationships between the real net peak currents on hemispherical microelectrodes, with the surface area $=2 \pi r_{\mathrm{m}}^{2}$, and the electrode radius and frequency. For instance, if $\rho>0.2$ and $k_{\text {acc }}=1 \times 10^{4}$, this dependence is given by the equation:

$$
\Delta I_{\mathrm{p}}=2 \pi n F c_{\mathrm{Ox}}^{*} r_{\mathrm{m}} \sqrt{D}\left(6.4 r_{\mathrm{m}} \sqrt{f}+22.5 \sqrt{D}\right)
$$

Assuming that $D=9 \times 10^{-6} \mathrm{~cm}^{2} \mathrm{~s}^{-1}$ and $f=100 \mathrm{~Hz}$, two terms in the brackets of Eq. (12) are equal if $r_{\mathrm{m}}=$ $10.55 \mu \mathrm{m}$. The calculations show that the contribution of transient (frequency dependent) component is decreased as the electrode radius is diminished and the duration of accumulation is increased. The steady-state (frequency independent) stripping peak current calculated from Eq. (12) is given by the following equation: $\Delta I_{\mathrm{p}}=45 \pi n F c_{\mathrm{Ox}}^{*} r_{\mathrm{m}} D$.

Figure $5 \mathrm{~b}$ shows that the peak potentials are practically independent of the parameter $k_{\text {acc }}$. The straight lines are the first approximations that are defined by the equations $E_{\mathrm{p}}-E^{\circ}=-0.30 \rho+0.003(\mathrm{~V})$ and $E_{\mathrm{p}}-E^{\circ}=$ $-0.13 \rho-0.032(\mathrm{~V})$, respectively.

In these calculations the low solubility of metal atoms in mercury is ignored and the theory applies to ions in traces only. 

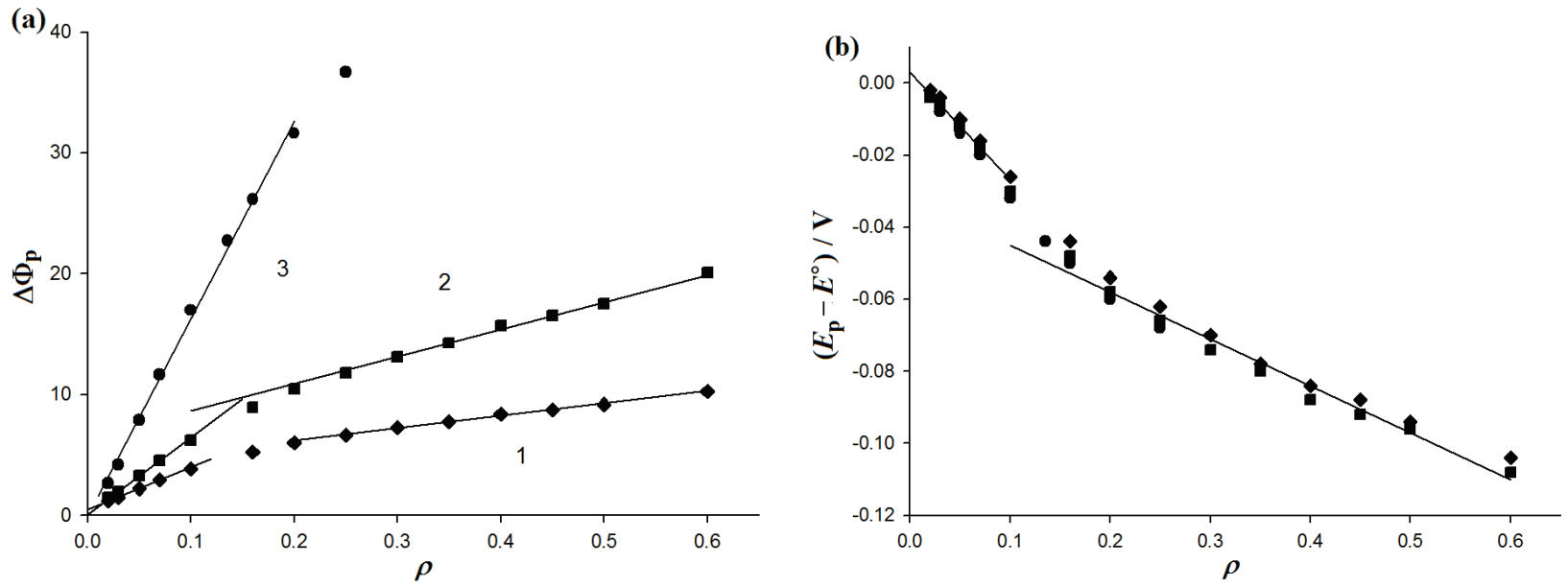

Figure 5. Dependence of anodic stripping square wave voltammetric (a) net peak current; and (b) peak potential on the dimensionless inverse electrode radius; (1) $k_{\mathrm{acc}}=2 \times 10^{3}$; (2) $k_{\mathrm{acc}}=1 \times 10^{4}$; and (3) $k_{\mathrm{acc}}=5 \times 10^{4}$. All other parameters are as in Figure 1 .

\section{CONCLUSION}

Anodic stripping square-wave voltammetry on spherical microelectrodes is characterized by near steady-state conditions. The influence of spherical diffusion is higher if the accumulation is longer and electrode radius is smaller. Because of restricted diffusion field within mercury drop, the maxima and minima of oxidative and reductive components of the response do not vanish as the radius is diminished. The real stripping peak current depends linearly on the square root of frequency, with the intercept that is equal to the steady-state current.

Acknowledgements. The financial support by the Croatian Science Foundation in the frame of the project number IP-112013-2072 is gratefully acknowledged.

\section{REFERENCES}

1. J. Hasegawa, S. Uno, and K. Nakazato, Jpn. J. Appl. Phys. 50 (2011) 04DL03-1-04DL03-9.

2. M.-L. Tercier-Waeber and M. Taillefert, J. Environ. Monit. 10 (2008) 30-54.

3. M. Koudelka-Hep and P. D. van der Wal, Electrochim. Acta 45 (2000) 2437-2441.

4. F. Davis and S. P. J. Higson, Environ. Sci.: Processes Impacts $\mathbf{1 5}$ (2013) 1477-1489.

5. C. M. Li and W. Hu, J. Electroanal. Chem. 688 (2013) 20-31.

6. A. Economou and P. R. Fielden, Anal. Chim. Acta 305 (1995) $165-175$.

7. H. Z. Brainina, Anal. Chim. Acta 305 (1995) 146-153.

8. C. Kokkinos, A. Economou, J. Raptis, and T. Speliotis,
Electrochem. Commun. 13 (2011) 391-395.

9. F. Marken, M. L. Gerrard, I. M. Mellor, R. J. Mortimer, C. E. Madden, S. Fletcher, K. Holt, J. S. Foord, R. H. Dahm, and F. Page, Electrochem. Commun. 3 (2001) 177-180.

10. J. Wang, S. B. Hočevar, R. P. Deo, and B. Ogorevc, Electrochem. Commun. 3 (2001) 352-356.

11. L. Rajendran, Electrochem. Commun. 2 (2000) 531-534.

12. A. Molina, C. Serna, F. Martinez-Ortiz, and E. Laborda, Electrochem. Commun. 10 (2008) 376-381.

13. G. Schulze and W. Frenzel, Anal. Chim. Acta 159 (1984) 95103.

14. S. L. R. Harvey, K. H. Parker, and D. O'Hare, J. Electroanal. Chem. 610 (2007) 122-130.

15. C. Cachet-Vivier, M. Keddam, V. Vivier, and L. T. Yu, $J$. Electroanal. Chem. 688 (2013) 12-19.

16. C. Belmont, M.-L. Tercier, J. Buffle, G. C. Fiaccabrino, and M. Koudelka-Hep, Anal. Chim. Acta 329 (1996) 203-214.

17. A. M. Bond and F. Scholz, Z. Chem. 30 (1990) 117-129.

18. H.-J. Kim, K.-S. Yun, E. Yoon, and J. Kwak, Electrochim. Acta 50 (2004) 205-210.

19. S. P. Kounaves, J. J. O'Dea, P. Chandrasekhar, and J. Osteryoung, Anal. Chem. 59 (1987) 386-389.

20. M. Rudolph, J. Electroanal. Chem. 503 (2001) 15-27.

21. S. Daniele, M. A. Baldo, M. Corbetta, and G. A. Mazzocchin, J. Electroanal. Chem. 379 (1994) 261-270.

22. M. A. Baldo, S. Daniele, and G. A. Mazzocchin, Electrochim. Acta 41 (1996) 811-818.

23. S̆. Komorsky-Lovrić, D. Jadreško and M. Lovrić, Electrochim. Acta 130 (2014) 286-289.

24. S. W. Feldberg, in A. J. Bard (ed.), Electroanalytical Chemistry, Vol. 3, Marcel Dekker, New York, 1969, p. 199-296.

25. D. Jadreško, M. Zelić and M. Lovrić, J. Electroanal. Chem. 645 (2010) 103-108.

26. $\breve{S}$. Komorsky-Lovrić, in F. Scholz (Ed.), Electroanalytical Methods, $2^{\text {nd }}$ edition, Springer, Berlin 2010, p. 273-290. 